\title{
Front Matter: Volume 7748
}

, "Front Matter: Volume 7748," Proc. SPIE 7748, Photomask and NextGeneration Lithography Mask Technology XVII, 774801 (15 June 2010); doi: $10.1117 / 12.869338$

SPIE. Event: Photomask and NGL Mask Technology XVII, 2010, Yokohama, Japan 


\section{PROCEEDINGS OF SPIE}

\section{Photomask and Next-Generation Lithography Mask Technology XVII}

Kunihiro Hosono

Editor

13-15 April 2010

Yokohama, Japan

Sponsored by

PMJ Photomask Japan

BACUS

SPIE

Published by

SPIE 
The papers included in this volume were part of the technical conference cited on the cover and title page. Papers were selected and subject to review by the editors and conference program committee. Some conference presentations may not be available for publication. The papers published in these proceedings reflect the work and thoughts of the authors and are published herein as submitted. The publisher is not responsible for the validity of the information or for any outcomes resulting from reliance thereon.

Please use the following format to cite material from this book:

Author(s), "Title of Paper," in Photomask and Next-Generation Lithography Mask Technology XVII, edited by Kunihiro Hosono, Proceedings of SPIE Vol. 7748 (SPIE, Bellingham, WA, 2010) Article CID Number.

ISSN 0277-786X

ISBN 9780819482389

Published by

SPIE

P.O. Box 10, Bellingham, Washington 98227-0010 USA

Telephone +1 3606763290 (Pacific Time) · Fax +1 3606471445

SPIE.org

Copyright (C) 2010, Society of Photo-Optical Instrumentation Engineers

Copying of material in this book for internal or personal use, or for the internal or personal use of specific clients, beyond the fair use provisions granted by the U.S. Copyright Law is authorized by SPIE subject to payment of copying fees. The Transactional Reporting Service base fee for this volume is $\$ 18.00$ per article (or portion thereof), which should be paid directly to the Copyright Clearance Center (CCC), 222 Rosewood Drive, Danvers, MA 01923. Payment may also be made electronically through CCC Online at copyright.com. Other copying for republication, resale, advertising or promotion, or any form of systematic or multiple reproduction of any material in this book is prohibited except with permission in writing from the publisher. The CCC fee code is $0277-786 \mathrm{X} / 10 / \$ 18.00$.

Printed in the United States of America.

Publication of record for individual papers is online in the SPIE Digital Library.

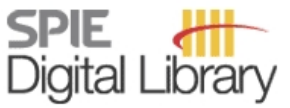

SPIEDigitalLibrary.org

Paper Numbering: Proceedings of SPIE follow an e-First publication model, with papers published first online and then in print and on CD-ROM. Papers are published as they are submitted and meet publication criteria. A unique, consistent, permanent citation identifier (CID) number is assigned to each article at the time of the first publication. Utilization of CIDs allows articles to be fully citable as soon they are published online, and connects the same identifier to all online, print, and electronic versions of the publication. SPIE uses a six-digit CID article numbering system in which:

- The first four digits correspond to the SPIE volume number.

- The last two digits indicate publication order within the volume using a Base 36 numbering system employing both numerals and letters. These two-number sets start with 00, 01, 02, 03, 04, $05,06,07,08,09,0 A, 0 B \ldots 0 Z$, followed by 10-1Z, 20-2Z, etc.

The CID number appears on each page of the manuscript. The complete citation is used on the first page, and an abbreviated version on subsequent pages. Numbers in the index correspond to the last two digits of the six-digit CID number. 


\section{Contents}

xi Conference Committees

INVITED PAPERS SESSION

774802 Perspectives of CMOS technology and future requirements (Keynote Paper) [7748-09]

T. Mogami, Semiconductor Leading Edge Technologies, Inc. (Japan)

774803 Actinic EUVL mask blank inspection capability with time delay integration mode (Invited Paper) [7748-83]

T. Yamane, T. Tanaka, T. Terasawa, O. Suga, Semiconductor Leading Edge Technologies, Inc. (Japan)

EUV MASK I

774804 Effect of EUV exposure upon surface residual chemicals on EUV mask surface [7748-93] H.-S. Lee, J. Choi, D. Lee, H. Ko, S. Kim, C.-U. Jeon, H. Cho, SAMSUNG Electronics Co., Ltd. (Korea, Republic of)

774805 Thin absorber EUV mask with light-shield border of etched multilayer and its lithographic performance [7748-72]

T. Kamo, H. Aoyama, Y. Arisawa, K. Tawarayama, T. Tanaka, O. Suga, Semiconductor Leading Edge Technologies, Inc. (Japan)

\section{EUV MASK II}

774806 Fast and highly accurate simulation of the printing behavior of EUV multilayer defects based on different models [7748-52]

F. Shao, Fraunhofer Institute for Integrated Systems and Device Technology (Germany) and Univ. of Erlangen-Nuremberg (Germany); P. Evanschitzky, K. Motzek, Fraunhofer Institute for Integrated Systems and Device Technology (Germany); A. Erdmann, Fraunhofer Institute for Integrated Systems and Device Technology (Germany) and Univ. of Erlangen-Nuremberg (Germany)

774807 Defect inspection and repair performance comparisons between EUV and conventional masks [7748-71]

K. Seki, M. Shibita, T. Yoshii, T. Fujimoto, Y. Sakata, S. Akima, Toppan Printing Co., Ltd. (Japan)

774808 Inspecting EUV mask blanks with a 193-nm system [7748-37]

J. Glasser, S. Stokowski, G. Inderhees, KLA-Tencor Corp. (United States) 
$774809 \quad$ Fabrication of ridge-and-groove servo pattern consisting of self-assembled dots for high-density bit patterned media (Invited Paper) [7748-03]

Y. Kamata, A. Kikitsu, N. Kihara, S. Morita, K. Kimura, H. Izumi, Toshiba Corp. (Japan)

7748 OA Defect reduction of patterned media templates and disks [7748-59]

K. Luo, S. Ha, J. Fretwell, R. Ramos, Z. Ye, G. Schmid, D. LaBrake, D. J. Resnick,

S. V. Sreenivasan, Molecular Imprints, Inc. (United States)

\section{MATERIAL AND PROCESS}

$77480 \mathrm{C}$ The relationship between mounting pressure and time on final photomask flatness [7748-31]

T. Mizoguchi, Toppan Photomasks, Inc. (United States); M. Barrett, IBM Corp. (United States);

S. Akutagawa, Toppan Photomasks, Inc. (United States); M. Caterer, R. Nolan, D. Plouffe,

N. Zhou, IBM Corp. (United States)

7748 OD Study of etching process for LER and resolution [7748-51]

T. Imoto, Y. Kojima, N. Fukugami, T. Haraguchi, T. Tanaka, Toppan Printing Co., Ltd. (Japan)

\section{PROCESS AND REPAIR}

7748 OE Preserving the mask integrity for the lithography process [7748-40]

S. Singh, HamaTech USA, Inc. (United States); P. Dress, HamaTech APE (Germany); U. Dietze, HamaTech USA, Inc. (United States)

7748 OF Haze risk reduced mask manufacturing process [7748-19]

P. Nesladek, V. Baudiquez, E. Foca, B. Sass, Advanced Mask Technology Ctr. GmbH Co. KG (Germany)

7748 OG Through-pellicle defect repair for advanced photomasks [7748-33]

T. Robinson, R. White, R. Bozak, J. LeClaire, M. Archuletta, D. Lee, RAVE LLC (United States)

\section{PATTERN GENERATION}

$7748 \mathrm{OH}$ eMET: development of a $50 \mathrm{keV}$ electron projection multibeam mask exposure tool for the $16 \mathrm{~nm} \mathrm{hp}$ technology node and below [7748-47]

E. Platzgummer, S. Cernusca, C. Klein, S. Kvasnica, B. Sonalkar, H. Loeschner, IMS

Nanofabrication AG (Austria)

7748 Ol Evaluation of a next generation EB mask writer for hp 32nm lithography [7748-55] T. Komagata, T. Hasegawa, K. Goto, JEOL Ltd. (Japan); K. Kono, R. Yamamoto, N. Nishida, HOYA Corp. (Japan); Y. Nakagawa, JEOL Ltd. (Japan)

7748 0J Mask writing time explosion and its effect on CD control in e-beam lithography [7748-92] S. H. Lee, J. Choi, S. J. Min, H. B. Kim, B. G. Kim, S.-G. Woo, H.-K. Cho, SAMSUNG Electronics Co., Ltd. (Korea, Republic of) 
7748 OK The fusion of metrology and inspection: challenges and solutions (Invited Paper) [7748-58] M. Higuchi, Toppan Printing Co., Ltd. (Japan)

$7748 \mathrm{OL} \quad$ Advanced mask-to-mask overlay analysis for next generation technology node reticles [7748-68]

K.-D. Roeth, F. Laske, K.-H. Schmidt, D. Adam, O. Ache, D. Ilsen, KLA-Tencor MIE GmbH (Germany)

7748 OM PROVE, the next generation registration metrology tool: status report [7748-77]

D. Beyer, Carl Zeiss SMS GmbH (Germany); P. Gabella, G. Hughes, SEMATECH North (United States); G. Klose, Carl Zeiss SMT AG (Germany); N. Rosenkranz, Carl Zeiss SMS GmbH (Germany)

7748 ON New critical dimension uniformity measurement concept based reticle inspection tool [7748-18]

K. Seo, M. Kim, S. C. Kim, J. Shin, C. Kim, Hynix Semiconductor Inc. (Korea, Republic of); J. Miller, A. Dayal, T. Hutchinson, KLA-Tencor Corp. (United States); K. Park, KLA-Tencor Corp. (Korea, Republic of)

$774800 \quad$ Evaluation of metrology capabilities of mask inspection equipment [7748-63]

T. Touya, S. Tamamushi, N. Takamatsu, NuFlare Technology Inc. (Japan)

7748 OP Computational inspection applied to a mask inspection system with advanced aerial imaging capability [7748-88]

A. Sagiv, A. Tam, W. Staud, Applied Materials, Inc. (Israel); L. Pang, D. Peng, L. He, D. Chen, T. Dam, V. Tolani, Luminescent Technologies, Inc. (United States)

$77480 Q \quad$ Productivity improvement of high resolution inspection mode on TeraScan 597XR [7748-85] H. Imai, T. Kosuge, K. Mesuda, E. Tsujimoto, H. Takamizawa, Dai Nippon Printing Co., Ltd. (Japan)

\section{MASK-RELATED LITHOGRAPHY}

7748 OR Extension of optical lithography by mask-litho integration with computational lithography (Invited Paper) [7748-57]

T. Takigawa, Brion Technologies KK (Japan); K. Gronlund, J. Wiley, Brion Technologies, Inc. (United States)

7748 OS Development of computational spacer patterning technology [7748-101]

H. Mashita, T. Taguchi, F. Nakajima, Toshiba Corp. (Japan); K. Iyanagi, Semiconductor Leading Edge Technologies, Inc. (Japan); T. Kotani, S. Mimotogi, S. Inove, Toshiba Corp. (Japan)

7748 OT Design compliant source mask optimization (SMO) [7748-39]

R. Socha, ASML (United States); T. Jhaveri, PDF Solutions (United States); M. Dusa, ASML (United States); X. Liu, L. Chen, S. Hsu, Z. Li, ASML Brion (United States); A. J. Strojwas, PDF Solutions (United States) 
7748 OU Point-source approach of source-mask optimization [7748-36]

W. Gao, Synopsys Inc. (Germany); S. Sethi, Synopsys Inc. (United States); V. Domnenko, Synopsys Inc. (Russian Federation); W. Hoppe, W. Demmerle, Synopsys Inc. (Germany); K. Lucas, Synopsys Inc. (United States)

7748 OV Best depth of focus on 22-nm logic wafers with less shot count [7748-50]

A. Fujimura, D2S, Inc. (United States); D. Kim, Luminescent Technologies, Inc. (United States); T. Komagata, Y. Nakagawa, JEOL Ltd. (Japan); V. Tolani, T. Cecil, Luminescent Technologies, Inc. (United States)

EDA, DFM, AND MDP

7748 OX Writing wavy metal 1 shapes on 22-nm logic wafers with less shot count [7748-60] H. R. Zable, A. Fujimura, D2S, Inc. (United States); T. Komagata, Y. Nakagawa, JEOL Ltd. (Japan); J. S. Petersen, Petersen Advanced Lithography (United States)

7748 OY Extraction and utilization of the repeating patterns for CP writing in mask making [7748-25] M. Shoji, T. Inove, M. Yamabe, Association of Super-Advanced Electronics Technologies (Japan)

\section{POSTER SESSION: MATERIAL AND PROCESS}

$77480 Z$ Two-fluid cleaning technology for advanced photomask [7748-84]

Y. Nagashima, K. Higuchi, T. Kikuchi, Y. Kurokawa, H. Hirose, M. Nonaka, Shibaura Mechatronics Corp. (Japan)

$774810 \quad$ Evaluation of easily removable pellicle adhesive [7748-32]

N. Zhou, M. Barrett, R. Nolan, D. Plouffe, J. Ritter, A. Wagner, M. Caterer, IBM Corp. (United States); T. Mizoguchi, S. Akutagawa, Toppan Photomasks, Inc. (United States); K. Duong, Micro Lithography, Inc. (United States); C. Imai, Zysan Corp. (United States); C. B. Wang, Micro Lithography, Inc. (United States)

$774811 \quad$ New type of haze formation on masks fabricated with Mo-Si blanks [7748-29] E. Foca, Advanced Mask Technology Ctr. GmbH Co. KG (Germany); A. Tchikoulaeva, GLOBALFOUNDRIES Dresden Module Two GmbH \& Co. KG (Germany); B. Sass, Advanced Mask Technology Ctr. GmbH Co. KG (Germany); C. West, Toppan Photomasks, Inc. (United States); P. Nesladek, Advanced Mask Technology Ctr. GmbH Co. KG (Germany); R. Horn, Toppan Photomasks Inc. (Germany)

774812 Pinhole defect study and process optimization [7748-80]

W.-H. Chae, G.-H. Hwang, S.-K. Seo, D.-H. Kim, D.-H. Lee, M.-H. Choi, S.-S. Choi, PKL Co., Ltd. (Korea, Republic of); D.-G. Kim, D.-H. Geum, G.-S. Nam, SNS TECH Corp. (Korea, Republic of)

774813 Evolution of etch profile and CD variation in mask making and SPT: simulations using TRAVIT software [7748-90]

S. Babin, K. Bay, Abeam Technologies, Inc. (United States) 
774814 Performance and stability of mask process correction for EBM-7000 [7748-81]

Y. Saito, Brion Technologies KK (Japan); G. Chen, J.-S. Wang, S. Bai, R. Howell, J. Li, J. Tao, D. VanDenBroeke, J. Wiley, Brion Technologies, Inc. (United States); T. Takigawa, Brion Technologies KK (Japan); T. Ohnishi, T. Kamikubo, S. Hara, H. Anze, Y. Hattori, S. Tamamushi, NuFlare Technology, Inc. (Japan)

$774815 \quad$ W-CMOS blanking device for projection multibeam lithography [7748-35] M. Jurisch, M. Irmscher, F. Letzkus, IMS Chips (Germany); S. Eder-Kapl, C. Klein, H. Loeschner, W. Piller, E. Platzgummer, IMS Nanofabrication AG (Austria)

774816 Evaluation of throughput improvement and character projection in multi-column-cell e-beam exposure system [7748-26]

A. Yamada, Y. Oae, T. Okawa, M. Takizawa, M. Yamabe, Association of Super-Advanced Electronics Technologies (Japan)

774817 Monitor technology of outer circumstances for mask EB writing system [7748-06] H. Hoshi, N. Samoto, H. Manabe, O. Wakimoto, S. lida, M. Yamabe, Association of Super-Advanced Electronics Technologies (Japan)

774818 Practical resist model calibration for e-beam direct write processes [7748-54] M. Schulz, H.-J. Stock, U. Klostermann, W. Hoppe, Synopsys GmbH (Germany); L. Bomholt, Synopsys Switzerland LLC (Switzerland); P. Jaschinsky, K.-H. Choi, M. Gutsch, Fraunhofer-Ctr. Nanoelektronische Technologien (Germany); H. Sailer, S. Martens, IMS CHIPS (Germany)

774819 Requirements of e-beam size and position accuracy for photomask of sub-32-nm HP device [7748-91]

J. Choi, S. H. Lee, H. B. Kim, B. G. Kim, S.-G. Woo, H. K. Cho, SAMSUNG Electronics Co., Ltd. (Korea, Republic of)

\section{POSTER SESSION: METROLOGY AND INSPECTION}

77481 A The large contour data generation from divided image of photomask pattern of $32 \mathrm{~nm}$ and beyond [7748-42]

T. Murakawa, Y. Ogiso, T. Iwai, J. Matsumoto, T. Nakamura, Advantest Corp. (Japan)

7748 1B Study on counting error in particle inspection [7748-78]

M. Amemiya, K. Ota, T. Taguchi, O. Suga, MIRAI-Semiconductor Leading Edge Technologies, Inc. (Japan)

7748 1C Assessing equipment and process related electrostatic risks to reticles with E-Reticle system [7748-53]

R. Tu, Benchmark Technologies Inc. (United States); T. Sebald, ESTION GmbH \& Co. KG (Germany)

7748 ID Large scale flash memory system (LSFMS) for photomask defect inspection machine [7748-21]

S. Yamamoto, Agile Patch Solutions Inc. (Japan); R. Pai, M. Ranade, S. Mondal, S. Prabhu, SoftJin Technologies Pvt. Ltd. (India); G. Kurosaki, Selcon Technology Inc. (Japan) 
$7748 \mathrm{IE} \quad$ Compensating for image placement errors for the HP $\mathbf{3 X} \mathbf{n m}$ node [7748-200]

E. S. Park, S. P. Kim, T. J. Ha, C. R. Kim, Hynix Semiconductor Inc. (Korea, Republic of)

7748 IF Increased productivity of repair verification by offline analysis of aerial images [7748-02]

E. Villa, L. Sartelli, H. Miyashita, DNP Photomask Europe (Italy); T. Scheruebl, R. Richter,

T. Thaler, Carl Zeiss SMS GmbH (Germany)

7748 1G Mask inspection system with variable sensitivity and printability verification function [7748-28]

T. Inove, K. Takahara, H. Tsuchiya, M. Tokita, T. Inove, M. Yamabe, Association of

Super-Advanced Electronics Technologies (Japan)

\section{POSTER SESSION: REPAIR}

$77481 \mathrm{H} \quad$ Nanomachining of non-orthogonal mask patterns [7748-34]

T. Robinson, D. Yi, R. White, R. Bozak, M. Archuletta, D. Lee, RAVE LLC (United States)

$774811 \quad$ FIB-CVD technology for EUV mask repair [7748-64]

T. Amano, N. Takagi, H. Shigemura, T. Terasawa, O. Suga, MIRAI-Semiconductor Leading

Edge Technologies, Inc. (Japan); K. Shiina, F. Aramaki, T. Kozakai, O. Matsuda, A. Yasaka, SII

NanoTechnology Inc. (Japan)

$7748 \mathrm{lJ} \quad$ Future application of e-beam repair tool beyond $3 \mathrm{X}$ generation [7748-11]

S. Kanamitsu, T. Hirano, Toshiba Corp. (Japan)

POSTER SESSION: EDA, DFM, AND MDP

$77481 \mathrm{~L} \quad$ A novel pattern error detecting algorithm for SEM images of mask monitoring patterns [7748-45]

Y. Oh, J.-P. Shin, J. Choi, J.-B. Lee, M.-H. Yoo, SAMSUNG Electronics Co., Ltd. (Korea, Republic of)

$77481 \mathrm{M} \quad$ CMP dummy pattern based on VSB writer load [7748-15]

W. Sakata, I. Osawa, S. Narukawa, T. Takikawa, H. Mohri, Dai Nippon Printing Co., Ltd. (Japan)

7748 iN Evaluation of mask manufacturing efficiency using mask data rank information [7748-08]

K. Kato, M. Endo, T. Inove, M. Yamabe, Association of Super-Advanced Electronics

Technologies (Japan); S. Nakatake, Kitakyushu Univ. (Japan)

774810 Efficient OASIS.MASK reader [7748-41]

D. Morales, J. P. Canepa, D. Cohen, Synopsys, Inc. (Chile)

7748 IP Efficiently writing circular contacts on production reticle [7748-49]

A. Fujimura, D2S, Inc. (United States); C. Pierrat, IC Images Technologies, Inc. (United States);

T. Kiuchi, T. Komagata, Y. Nakagawa, JEOL Ltd. (Japan)

7748 IR Machine specific fracture optimization for JEOL e-beam mask writer [7748-20]

J. Yeap, R. Kondepudy, P. Kulkarni, Synopsys, Inc. (United States); Y. Kawase, R. Cinque, JEOL Ltd. (Japan) 
7748 is Contact mask LER impact on lithographic performance [7748-48]

T. Nagatomo, M. Yamana, K. Morinaga, M. Higuchi, S. Sato, T. Tanaka, Toppan Printing Co., Ltd. (Japan)

7748 IT E-beam writing time improvement for inverse lithography technology mask for full-chip [7748-86]

G. Xiao, D. H. Son, T. Cecil, D. Irby, D. Kim, K.-H. Baik, Luminescent Technologies, Inc. (United States); B.-G. Kim, S. Jung, S. S. Suh, H. Cho, Samsung Electronics Co. Ltd. (Korea, Republic of)

7748 IU Aberration-aware robust mask design with level-set-based inverse lithography [7748-05] Y. Shen, N. Wong, E. Y. Lam, The Univ. of Hong Kong (Hong Kong, China)

7748 IV Improvement of KrF contact layer by inverse lithography technology with assist feature [7748-96]

S. Jun, Y.-A. Shim, J. Choi, K. Choi, J. Han, Dongbu HiTek Co., Ltd. (Korea, Republic of); K. Wang, J. McCarthy, G. Xiao, G. Dai, D. Son, X. Zhou, T. Cecil, D. Kim, K. Baik, Luminescent Technologies, Inc. (United States)

\section{POSTER SESSION: NGL MASKS AND APPLICATIONS}

7748 1X The optical CD metrology for EUV mask [7748-94]

J.-B. Park, K.-Y. Bang, D.-G. Lee, H.-Y. Jeong, S.-S. Kim, H.-K. Cho, SAMSUNG Electronics Co., Ltd. (Korea, Republic of)

7748 IY EUV mask inspection with 193-nm inspector for 32- and 22-nm HP [7748-10] D. Wack, Q. Q. Zhang, G. Inderhees, D. Lopez, KLA-Tencor Corp. (United States)

$77481 \mathrm{Z}$ Challenges for quality $15 \mathrm{~nm}$ groove patterning with ZEP520A for a master fabrication for track pitch 50nm full-surface DTR-Media [7748-202]

H. Iyama, K. Hamamoto, S. Kishimoto, M. Nakano, T. Kagatsume, T. Sato, H. Kobayashi,

T. Watanabe, HOYA Corp. (Japan)

774820 Evaluation of transfer of particles from dual-pod base plate to EUV mask [7748-62] M. Yonekawa, K. Ota, T. Taguchi, O. Suga, MIRAl-Semiconductor Leading Edge Technologies, Inc. (Japan)

$774821 \quad$ Characterization of line-width roughness about 22-32nm node EUV mask [7748-75] Y. Inazuki, T. Abe, T. Takikawa, H. Mohri, N. Hayashi, Dai Nippon Printing Co., Ltd. (Japan)

774822 Evaluation of novel EUV mask inspection technologies [7748-89]

S. Mangan, A. Kantor, N. Shoshani, A. Jaffe, D. Kasimov, V. Kudriashov, R. Brikman, L. Shoval, A. Sreenath, Applied Materials (Israel)

774823 Short-range electron backscattering from EUV masks [7748-01]

H. Tanabe, Intel K.K. (Japan); T. Abe, Y. Inazuki, N. Hayashi, Dai Nippon Printing Co. Ltd. (Japan) 
774824 Effective-exposure-dose monitoring technique in EUV lithography [7748-99]

Y. Nakajima, K. Kasa, T. Sato, M. Asano, S. Kyoh, Toshiba Corp. (Japan); H. Mizuno, Toshiba America Electronic Components (United States)

774825 Fine pixel SEM image for EUV mask pattern 3D quality assurance based on lithography simulation [7748-201]

E. Yamanaka, M. Itoh, Toshiba Corp. (Japan); M. Kato, K. Ueno, K. Hayashi, A. Higuchi, Topcon Corp. (Japan); N. Hayashi, Dai Nippon Printing Co., Ltd. (Japan)

774826 Challenges in flare correction in EUVL lithography for half pitch 22-nm generation [7748-76] T. Uno, Y. Arisawa, H. Aoyama, T. Tanaka, Semiconductor Leading Edge Technologies, Inc. (Japan)

\section{POSTER SESSION: METROLOGY AND INSPECTION (CONTINUED)}

774827 Photomask defect detection and inspection: aerial imaging and high resolution inspection strategies [7748-203]

S. Oh, D. Hwang, I. Kim, C. Kim, Hynix Semiconductor Inc. (Korea, Republic of); A. Tam, M. Ben Yishai, Y. Wolff, Applied Materials (Israel)

774828 Results from a new 193nm die-to-database reticle inspection platform [7748-95]

W. H. Broadbent, D. S. Alles, M. T. Giusti, D. F. Kvamme, R.-F. Shi, W. L. Sousa, R. Walsh, Y. Xiong, KLA-Tencor Corp. (United States)

Author Index 


\title{
Conference Committees
}

\author{
Symposium Chair
}

Toshiyuki Horiuchi, Tokyo Denki University (Japan)

Symposium Vice Chair

Masato Shibuya, Tokyo Polytechnic University (Japan)

Advisory Committee Chair

Yasuo Tarui, Tokyo University of Agriculture and Technology (Japan)

Members

Masanori Komuro, New Energy and Industrial Technology

Development Organization (Japan)

Masatoshi Migitaka, Toyota Technological Institute (Japan)

Masao Otaki, Toppan Printing Company, Ltd. (Japan)

Norio Saito, Nippon Institute of Technology (Japan)

Yoshio Tanaka, Luminescent Technologies Inc. (Japan)

Organizing Committee Chair

Vice Chair

Toshiyuki Horiuchi, Tokyo Denki University (Japan)

Masato Shibuya, Tokyo Polytechnic University (Japan)

Members

Uwe Behringer, UBC Microelectronics (Germany)

Parkson Chen, Taiwan Mask Corporation (Taiwan)

Han-ku Cho, SAMSUNG Electronics Company, Ltd. (Korea, Republic of)

Brian J. Grenon, Grenon Consulting, Inc. (United States)

Hideaki Hamada, NuFlare Technology Inc. (Japan)

Naoya Hayashi, Dai Nippon Printing Company, Ltd. (Japan)

Eiichi Hoshino, Nikon Corporation (Japan)

Kunihiro Hosono, Renesas Technology Corporation (Japan)

Greg Hughes, International SEMATECH, (United States)

Hiroichi Kawahira, Sony Corporation (Japan)

Warren Montgomery, International SEMATECH (United States)

Ichiro Mori, Semiconductor Leading Edge Technologies, Inc. (Japan)

Hiroaki Morimoto, Toppan Printing Company, Ltd. (Japan)

Osamu Nagarekawa, HOYA Corporation (Japan)

Kenichiro Okuda, SEMI Japan (Japan) 
Hiroyuki Shigemura, Semiconductor Leading Edge Technologies, Inc. (Japan)

Yoshiki Suzuki, KLA-Tencor Japan Ltd. (Japan)

Tadahiro Takigawa, Brion Technologies K.K. (Japan)

Koichiro Tsujita, Canon Inc. (Japan)

Wataru Wakamiya, JEOL Ltd. (Japan)

Masaki Yamabe, Association of Super-Advanced Electronics

Technologies (ASET) (Japan)

Anto Yasaka, SII NanoTechnology Inc. (Japan)

Nobuyuki Yoshioka, Renesas Technology Corporation (Japan)

Steering Committee Chair

Hiroyuki Shigemura, Semiconductor Leading Edge Technologies, Inc.

Vice Chairs (Japan)

Morihisa Hoga, Dai Nippon Printing Company, Ltd. (Japan)

Tsuneo Terasawa, Semiconductor Leading Edge Technologies, Inc. (Japan)

Members

Takayuki Abe, NuFlare Technology Inc. (Japan)

Naoya Hayashi, Dai Nippon Printing Company, Ltd. (Japan)

Kunihiro Hosono, Renesas Technology Corporation (Japan)

Naoyuki Ishiwata, Fujitsu Microelectronics Ltd. (Japan)

Ichiro Kagami, Sony Corporation (Japan)

Kokoro Kato, SII NanoTechnology Inc. (Japan)

Toshio Konishi, Toppan Printing Company, Ltd. (Japan)

Koichi Moriizumi, Lasertec Corporation (Japan)

Takeshi Nakajima, KLA-Tencor Japan Ltd. (Japan)

Masatoshi Oda, NTT-AT Nanofabrication Corporation (Japan)

Yasushi Ohkubo, HOYA Corporation (Japan)

Toshiyuki Takahashi, JEOL Ltd. (Japan)

Hiroyoshi Tanabe, Intel K.K. (Japan)

Yoji Tonooka, Toppan Printing Company, Ltd. (Japan)

Hidehiro Watanabe, Toshiba Corporation (Japan)

Kazuo Yokoyama, Cadence Design Systems, Japan (Japan)

Program Committee Chair

Kunihiro Hosono, Renesas Technology Corporation (Japan)

Vice Chair

Kokoro Kato, SII NanoTechnology Inc. (Japan)

Toshio Konishi, Toppan Printing Company, Ltd. (Japan) 
Shinji Akima, Toppan Printing Company, Ltd. (Japan)

Akihiko Ando, NEC Electronics Corporation (Japan)

Jeff Farnsworth, Intel Corporation (United States)

Thomas B. Faure, IBM Corporation (United States)

Shigeru Hirukawa, Nikon Corporation (Japan)

Koji Hosono, Fujitsu Microelectronics Ltd. (Japan)

Gregg Inderhees, KLA-Tencor Corporation (United States)

Curt A. Jackson, Toppan Photomasks, Inc. (United States)

Byung-Gook Kim, Samsung Electronics Company, Ltd.

(Korea, Republic of)

John Lin, Taiwan Semiconductor Manufacturing Company, Ltd.

(Taiwan)

Mark Ma, Photronics, Inc. (United States)

Hiroshi Mohri, Dai Nippon Printing Company, Ltd. (Japan)

Koji Murano, Toshiba Corporation (Japan)

Yoshikazu Nagamura, Renesas Technology Corporation (Japan)

Yoshinori Nagaoka, KLA-Tencor Japan Ltd. (Japan)

Yasutoshi Nakagawa, JEOL Ltd. (Japan)

Noriaki Nakayamada, NuFlare Technology Inc. (Japan)

Nobuyuki Nishiguchi, Semiconductor Technology Academic

Research Center (Japan)

Yuji Nonami, Panasonic Corporation (Japan)

S. Schulz, Mentor Graphics Corporation (United States)

Yoshiyuki Sekine, Canon Inc. (Japan)

Yasunari Sohda, Hitachi, Ltd. (Japan)

Osamu Suga, Semiconductor Leading Edge Technologies, Inc. (Japan)

Minoru Sugawara, Sony Corporation (Japan)

Koji Takagi, Applied Materials Japan, Inc. (Japan)

Tatsuya Tomita, Dai Nippon Printing Company, Ltd. (Japan)

Yoichi Usui, HOYA Corporation (Japan)

Session Chairs

Invited Papers Session

Kunihiro Hosono, Renesas Technology Corporation (Japan)

John Lin, Taiwan Semiconductor Manufacturing Company, Ltd.

(Taiwan)

EUV Mask I

Hiroshi Mohri, Dai Nippon Printing Company, Ltd. (Japan)

Osamu Suga, Semiconductor Leading Edge Technologies, Inc.

(Japan)

Banqive Wu, Applied Materials, Inc. (United States) 
EUV Mask II

Osamu Suga, Semiconductor Leading Edge Technologies, Inc. (Japan)

Hiroshi Mohri, Dai Nippon Printing Company, Ltd. (Japan)

Banqive Wu, Applied Materials, Inc. (United States)

Nano Imprint and Patterned Media

Morihisa Houga, Dai Nippon Printing Company, Ltd. (Japan)

Youichi Usui, HOYA Corporation (Japan)

Material and Process

Toshio Konishi, Toppan Printing Company, Ltd. (Japan)

Koji Murano, Toshiba Corporation (Japan)

Thomas B. Faure, IBM Corporation (United States)

Process and Repair

Toshio Konishi, Toppan Printing Company, Ltd. (Japan)

Koji Murano, Toshiba Corporation (Japan)

Thomas B. Faure, IBM Corporation (United States)

Pattern Generation

Noriaki Nakayamada, NuFlare Technology, Inc. (Japan)

Jeff Farnsworth, Intel Corporation (United States)

Metrology and Inspection

Tatsuya Tomita, Dai Nippon Printing Company, Ltd. (Japan)

Shinji Akima, Toppan Printing Company, Ltd. (Japan)

Byung Gook Kim, SAMSUNG Electronics Company, Ltd.

(Korea, Republic of)

Mask-related Lithography

Minoru Sugawara, Sony Corporation (Japan)

Steffen Schulze, Mentor Graphics Corporation (United States)

EDA, DFM, and MDP

Kokoro Kato, SII NanoTechnology Inc. (Japan)

Nobuyuki Nishiguchi, Semiconductor Technology Academic Research Center (Japan) 